\title{
Tolerância da mandioca a diferentes herbicidas aplicados em pós-emergência ${ }^{1}$
}

\section{Tolerance cassava to different herbicides applied in post-emergence}

\author{
Hugo Falkyner da Silva Bandeira ${ }^{2}$; José Maria Arcanjo Alves ${ }^{3}$; Paulo Roberto Ribeiro Rocha ${ }^{4}$; \\ Anderson Strucker ${ }^{5}$; Luciana Baú Trassato ${ }^{6}$
}

Resumo - A mandioca apresenta ciclo longo e crescimento inicial lento, o que facilita desenvolvimento das plantas daninhas que irão competir com a cultura. Objetivou-se avaliar a tolerância da mandioca, cultivar Aciolina, a diferentes herbicidas, na fase inicial de desenvolvimento da cultura. $\mathrm{O}$ experimento foi conduzido em casa de vegetação, em delineamento inteiramente casualizados com quatro repetições. Os tratamentos avaliados foram: haloxyfop (60 $\mathrm{g} \mathrm{ha}^{-1}$ i.a), lactofen (180 $\left.\mathrm{g} \mathrm{ha}^{-1} \mathrm{i} . \mathrm{a}\right)$, chorimuron-ethyl (17,5 $\left.\mathrm{g} \mathrm{ha}^{-1} \mathrm{i} . \mathrm{a}\right)$, bentazon (576 $\mathrm{g} \mathrm{ha}^{-1}$ i.a), bentazon + imazamox $\left(600+28 \mathrm{~g} \mathrm{ha}^{-1}\right.$ i.a), imazethapyr $\left(100 \mathrm{~g} \mathrm{ha}^{-1} \mathrm{i} . \mathrm{a}\right)$, fomesafen $\left(250 \mathrm{~g} \mathrm{ha}^{-1} \mathrm{i} . \mathrm{a}\right)$, quizalofop-P-ethyl (62,5 $\mathrm{g} \mathrm{ha}^{-1}$ i.a), lactofen + quizalofop-P-ethyl $\left(180+62,5 \mathrm{~g} \mathrm{ha}^{-1} \mathrm{i} . \mathrm{a}\right)$, fomesafen + quizalofop-P-ethyl $\left(250+62,5 \mathrm{~g} \mathrm{ha}^{-1} \mathrm{i} . \mathrm{a}\right)$, além de uma testemunha. Foram realizadas avaliações visuais de intoxicação, altura e número de folhas, massa seca foliar, da haste, raiz, massa seca total e área foliar. Os herbicidas bentazon, fomesafen e fomesafen + quizalofop-P-ethyl provocaram injúrias elevadas, porém não comprometeram o desenvolvimento das plantas. $\mathrm{O}$ herbicida imazethapyr interferiu no desenvolvimento da cultura reduzindo em 58,03\% a altura e em 35,87\% a massa seca total das plantas. Foram observadas deformações nas folhas novas com o tratamento bentazon + imazamox. O uso do lactofen + quizalofop-P-ethyl resultou na morte das plantas, o uso do lactofen, reduziu todas as variáveis estudadas. A cv. Aciolina mostrou tolerância aos herbicidas halox yfop, quizalofop-P-ethyl e chlorimuron-ethyl. Os herbicidas lactofen, imazethapyr, bentazon + imazamox e o lactofen + quizalofop-P-ethyl provocam danos severos a cultura da mandioca.

Palavras-chaves: controle químico; Manihot esculenta Crantz; intoxicação por herbicidas

\begin{abstract}
Cassava has a long cycle and slow initial growth, which facilitates development of weeds that will compete with the culture. It was aimed to evaluate the tolerance of cassava cultivar Aciolina, the different herbicides in the early stages of crop development. The experiment was conducted in a greenhouse, in a completely randomized design with four replications. The evaluated treatments were: haloxyfop (60 $\mathrm{g} \mathrm{ha}^{-1} \mathrm{a}$.i.), lactofen (180 $\mathrm{g} \mathrm{ha}^{-1}$ a.i.), chorimuron-ethyl (17.5 $\mathrm{g} \mathrm{ha}^{-1}$ a.i.), bentazon (576 $\mathrm{g} \mathrm{ha}^{-1}$ a.i.), bentazon + imazamox $\left(600+28 \mathrm{~g} \mathrm{ha}^{-1}\right.$ a.i. $)$, imazethapyr (100 $\mathrm{g} \mathrm{ha}^{-1}$ a.i.), fomesafen (250 $\mathrm{g} \mathrm{ha}^{-1}$ a.i.), quizalofop-P-ethyl (62.5 $\mathrm{g} \mathrm{ha}^{-1}$ a.i.), lactofen +

\footnotetext{
${ }^{1}$ Recebido para publicação em 01/07/2016 e aceito em 04/08/2016.

${ }^{2}$ Eng. Agr., MSc., Agência de Defesa Agropecuária do Estado de Roraima (ADERR), Boa Vista, Roraima, Brasil. Email: hugo_falkyner@ hotmail.com

${ }^{3}$ Professor Titular, Departamento de Fitotecnia, Agronomia, Centro de Ciências Agrárias, Universidade Federal de Roraima, Boa Vista, Roraima, Brasil. E-mail: arcanjoalves@oi.com.br

${ }^{4}$ Professor Adjunto, Departamento de Solos e Engenharia Agrícola, Centro de Ciências Agrárias, Universidade Federal de Roraima, Boa Vista, Roraima, Brasil. E-mail: paulo.rocha@ufrr.br

${ }^{5}$ Eng. Agr., MSc., Agência de Defesa Agropecuária do Estado de Roraima (ADERR), Boa Vista, Roraima, Brasil. Email: andersonstriicker@hotmail.com

${ }^{6}$ Eng. Agr., MSc., Agência de Defesa Agropecuária do Estado de Roraima (ADERR), Boa Vista, Roraima, Brasil. Email: luciana.trassato@ hotmail.com
} 
quizalofop-P-ethyl $\left(180+62.5 \mathrm{~g} \mathrm{ha}^{-1}\right.$ a.i. $)$, fomesafen + quizalofop-P-ethyl $\left(250+62.5 \mathrm{~g} \mathrm{ha}^{-1}\right.$ a.i. $)$, and a control. Were performed visual assessments of intoxication, plant height and number of leaves, dry mass of the leaves, stem, root dry mass total and leaf area. The herbicides bentazon, fomesafen and fomesafen + quizalofop-P-ethyl caused high injury but not affect the development of plants. The imazethapyr herbicide interfered in development of culture by reducing in $58.03 \%$ the height and $35.87 \%$ dry weight of plants. Deformations were observed in young leaves with treatment bentazon + imazamox. The use of lactofen + quizalofop-P-ethyl resulted in plant death, the use of lactofen significantly reduced all variables. Cassava, cv. Aciolina, showed tolerance to herbicides haloxyfop and quizalofop and chlorimuron-ethyl. The lactofen, imazethapyr, bentazon + imazamox and quizalofop + lactofen cause severe damage to the cassava crop.

Keywords: chemical control; Manihot esculenta Crantz; intoxication by herbicides

\section{Introdução}

A mandioca (Manihot esculenta Crantz) é uma espécie cultivada em todo o Brasil. Tem sua produção ligada à agricultura familiar, principalmente nas regiões Norte e Nordeste, assumindo um importante papel social e econômico (Schons et al., 2009; Albuquerque et al., 2014). A cultura tem importante papel na alimentação humana devido à alta produtividade de calorias em sua raiz tuberosa. Suas folhas possuem elevado teor de proteína, sendo uma alternativa para alimentação animal (Nunes et al., 2009).

O lento estabelecimento da lavoura de mandioca após o plantio facilita o rápido desenvolvimento das plantas daninhas. Com o solo exposto no início do ciclo, as plantas daninhas se estabelecem rapidamente e têm vantagens competitivas sobre a mandioca, sendo esse um dos principais problemas que reduz a produtividade e viabilidade econômica da cultura (Silva et al., 2012b; Cardoso et al., 2013).

A comunidade de plantas infestantes presentes, sua agressividade, o ambiente e a época da convivência em relação à cultura, são fatores que influenciam o grau de interferência das plantas daninhas sobre a cultura da mandioca (Moura, 2000). Estudos relatam que, em média, entre 25 a 120 dias após o plantio, corresponde ao período em que a lavoura de mandioca deve se manter livre de plantas daninhas, sendo considerado, o Período Crítico de Prevenção a Interferência (PCPI) (Johanns e
Contiero, 2006; Albuquerque et al., 2008; Biffe et al., 2010). A ausência de controle de plantas daninhas pode provocar reduções superiores a $90 \%$ no rendimento da cultura em função do tempo de convivência e da densidade das espécies infestantes (Silva et al., 2009).

O controle de plantas daninhas em pósemergência na cultura da mandioca, é de fundamental importância, porém é restrita a disponibilidade de herbicidas registrados para serem aplicados nessa fase do cultivo. Dentre os herbicidas registrados, apenas dois princípios ativos são indicados para aplicação na fase inicial em pós-emergência, sendo esses recomendados para o controle de Poáceas (monocotiledôneas) (Brasil, 2016). Este fato tem restringido ainda mais a utilização do manejo químico na lavoura, levando em conta a incidência de plantas de folha larga (Biffe et al., 2010; Albuquerque et al., 2014).

A incorporação do uso de herbicidas no manejo de plantas daninhas é necessária, uma vez que melhora a eficácia de controle e reduz os custos de produção (Machado et al., 2006). Entretanto, há carência de informações sobre a tolerância da mandioca a herbicidas aplicados em pós-emergência, em uso isolado ou em misturas em tanque. As misturas entre herbicidas com diferentes mecanismos de ação podem contribuir na redução da pressão de seleção de biótipos resistentes e aumentar a eficiência no manejo das plantas daninhas. Assim objetivou-se com esse trabalho avaliar a tolerância da mandioca, cultivar Aciolina, a 
diferentes herbicidas, na fase inicial de desenvolvimento da cultura.

\section{Material e Métodos}

A pesquisa foi conduzida em casa de vegetação, pertencente ao Centro de Ciências Agrárias, da Universidade Federal de Roraima (CCA-UFRR), em condições controladas de temperatura e umidade. Utilizou-se solo da camada 0-20 cm de um Latossolo Amarelo distrófico (Ladx), de textura média (areia: $663 \mathrm{~g}$ $\mathrm{kg}^{-1}$; argila: $252 \mathrm{~g} \mathrm{~kg}^{-1}$ e silte: $85 \mathrm{~g} \mathrm{~kg}^{-1}$ ).

A análise dos atributos químicos do solo apresentou os seguintes valores: $\mathrm{pH}\left(\mathrm{H}_{2} \mathrm{O}\right)$ de 4,6; Matéria orgânica de 9,3 $\mathrm{g} \mathrm{kg}^{-1}$; $\mathrm{P}$ (Mehlich 1): $0,45 \mathrm{mg} \mathrm{kg}^{-1} ; \mathrm{Ca}^{2+}: 0,1 \mathrm{cmol}_{\mathrm{Ckg}^{-1}} ; \mathrm{Mg}^{2+}: 0,02$ cmol $_{\mathrm{c} \mathrm{kg}}{ }^{-1} ; \mathrm{K}^{+}: 0,06$ cmol $_{\mathrm{c}} \mathrm{kg}^{-1} ; \mathrm{H}^{+}+\mathrm{AL}^{3+}: 2,39$ cmol $_{\mathrm{c}} \mathrm{kg}^{-1}$; $\mathrm{AL}^{3+}: 0,75$ cmol $_{\mathrm{c}} \mathrm{kg}^{-1}$; CTCt de 2,55 $\mathrm{cmol}_{\mathrm{c}} \mathrm{dm}^{-3}$; V de $6,3 \%$ e m de $82,4 \%$.

Após a coleta do solo, foi feita incubação por 30 dias, com o uso de calcário dolomítico PRNT 95\%, para corrigir a acidez e elevar a saturação de base a $60 \%$. Terminado o período de incubação foi preparado um substrato, com uma mistura de $75 \%$ de solo e $25 \%$ de esterco bovino curtido. Em seguida realizou-se $\mathrm{o}$ enchimento de vasos com capacidade volumétrica de $8 \mathrm{dm}^{3}$. Para adequação do substrato quanto à nutrição, foram aplicados em cada vaso, o equivalente a $40 \mathrm{~kg} \mathrm{ha}^{-1}$ de ureia, $40 \mathrm{~kg} \mathrm{ha}^{-1}$ de cloreto de potássio e $220 \mathrm{~kg} \mathrm{ha}^{-1}$ de superfosfato simples (Silva et al., 2012a). As irrigações foram feitas diariamente, com 15 minutos de duração, ocorrendo as $08 \mathrm{~h} 00 \mathrm{~min}$, $12 \mathrm{~h} 30 \mathrm{~min}$ e $16 \mathrm{~h} 15 \mathrm{~min}$, por sistema automático de microaspersão.

Os tratamentos consistiram no uso de oito herbicidas com diferentes princípios ativos, duas misturas de herbicidas indicados para o controle de plantas mono e dicotiledôneas e uma testemunha sem aplicação de herbicida, conforme descrito na Tabela 1 . O delineamento adotado foi inteiramente casualizado, com 11 tratamentos e quatro repetições. Cada vaso representou uma unidade experimental. No dia 14 de março foi efetuado o plantio de três manivas de $15 \mathrm{~cm}$ por vaso. Passado o período de germinação, foi realizado o desbaste deixando-se três plantas por vaso.

Tabela 1. Princípios ativos dos herbicidas pós-emergentes, produto comercial e respectivas dosagens aplicados em plantas de mandioca.

\begin{tabular}{|c|c|c|}
\hline Princípios ativos & Produto Comercial & Dose $\left(\mathrm{g} \mathrm{ha}^{-1} \text { de i.a. }\right)^{*}$ \\
\hline Haloxyfop & Verdict, $120 \mathrm{~g} \mathrm{~L}^{-1}$ de i.a, EC, Dow AgroSciences & 60 \\
\hline Lactofen & Cobra , $240 \mathrm{~g} \mathrm{~L}^{-1}$ de i.a, EC, Bayer & 180 \\
\hline Chlorimuron-ethyl & Staron , $250 \mathrm{~g} \mathrm{~kg}^{-1}$ de i.a, WG, CropChem & 17,5 \\
\hline Bentazon & Basagran, $480 \mathrm{~g} \mathrm{~L}^{-1}$ de i.a, SL, Basf & 576 \\
\hline Bentazon + imazamox & Amplo, $600 \mathrm{~g} \mathrm{~L}^{-1}$ de i.a $+28 \mathrm{~g} \mathrm{~L}^{-1}$ de i.a, SL, Basf & $600+28$ \\
\hline Imazethapyr & Imazethapyr Plus, $100 \mathrm{~g} \mathrm{~L}^{-1}$ de i.a, SL, Nortox & 100 \\
\hline Fomesafen & Flex, $250 \mathrm{~g} \mathrm{~L}^{-1}$ de i.a, SL, Syngenta & 250 \\
\hline Quizalofop-P-ethyl & Targa, $50 \mathrm{~g} \mathrm{~L}^{-1}$ de i.a, EC, Bayer & 62,5 \\
\hline Lactofen + quizalofop-P-ethyl & Cobra + Targa & $180+62,5$ \\
\hline Fomesafen + quizalofop-P-ethyl & Flex + Targa & $250+62,5$ \\
\hline Testemunha & - & - \\
\hline
\end{tabular}

A aplicação dos tratamentos ocorreu no dia 14 de abril de 2015, aos 30 dias após o plantio (DAP) da mandioca. Foi utilizado um pulverizador manual costal com pressão constante de $150 \mathrm{kPa}$, equipado com bicos contendo ponta tipo leque modelo TT-11002, a uma altura de $50 \mathrm{~cm}$ do alvo, com velocidade de $1 \mathrm{~m} \mathrm{~s}^{-1}$, e volume de calda de $180 \mathrm{~L} \mathrm{ha}^{-1}$.

As avaliações de altura de planta e número de folhas ocorreram aos $0 ; 7 ; 14 ; 21$ dias após a aplicação dos herbicidas (DAAH). Tais variáveis resultaram das médias das três plantas presentes em cada vaso. 
Foram feitas avaliações visuais de intoxicação da cultura aos $3 ; 6 ; 9 ; 12 ; 15 ; 18$ e 21 (DAAH), com a utilização de uma escala percentual de notas variando entre 0 (zero) a 100 (cem), em que 0 implicaria ausência de quaisquer injúrias e 100 a morte da planta (SBCPD, 1995). Os critérios usados para o estabelecimento das notas foram: inibição do crescimento, quantidade e uniformidade das injúrias, coloração das folhas, deformidades no limbo foliar e capacidade de rebrota (emissão de folhas novas).

Aos 21 DAAH, posterior à última mensuração de altura e número de folhas, foi realizado o corte das plantas na base da haste, separando-se folhas e haste, e as raízes foram lavadas para retirar o excesso de solo. Todas as amostras da parte aérea (haste, folhas) e raízes foram acondicionadas em sacos de papel devidamente identificados e levados para o Laboratório de Análises de Fitotecnia da Pósgraduação (CCA-UFRR). Posteriormente com o uso do aparelho CID Bio-Science ${ }^{\circledR}$ modelo CI 202, foi mensurado a área foliar de cada parcela. Em seguida as amostras foram secas em estufa com circulação forçada de ar, a $65^{\circ} \mathrm{C}$, até atingir peso constante para determinação da massa seca.
Nas variáveis, avaliação de intoxicação, altura da planta e número de folhas, para a análise estatística foi adotado o esquema em parcelas subdivididas, considerando os tratamentos (herbicidas) como fator primário, e o tempo como fator secundário. Os dados das variáveis: altura de planta, número de folhas, massa seca e área foliar, foram transformados em porcentagem relativa à testemunha. Posteriormente foram submetidos à análise de variância e as médias quando significativas foram agrupadas segundo critério de ScottKnott, a 5\% de probabilidade de erro, por intermédio do programa estatístico SISVAR 5.3 .

\section{Resultados e Discussão}

Na Tabela 2 são apresentados, os valores referentes à altura das plantas de mandioca. As plantas tratadas com os herbicidas haloxyfop e quizalofop-P-ethyl, durante o período avaliado, apresentaram resultados similares ao da testemunha. Os demais herbicidas causaram reduções na variável avaliada, com efeitos mais acentuados nos tratamentos lactofen + quizalofop-P-ethyl e imazethapyr.

Tabela 2. Altura das plantas de mandioca, em percentagem relativa à testemunha aos $0,7,14$ e 21 dias após a aplicação dos tratamentos.

\begin{tabular}{|c|c|c|c|c|c|c|c|c|}
\hline \multirow{3}{*}{ Tratamentos } & \multicolumn{8}{|c|}{ Altura da planta $(\%)$} \\
\hline & \multicolumn{8}{|c|}{ Dias após a aplicação dos herbicidas } \\
\hline & \multicolumn{2}{|l|}{0} & \multicolumn{2}{|c|}{7} & \multicolumn{2}{|c|}{14} & \multicolumn{2}{|c|}{21} \\
\hline Haloxyfop & 86,25 & $\mathrm{~A} \mathrm{a}$ & 90,51 & $\mathrm{~A} \mathrm{a}$ & 90,67 & $\mathrm{~A} \mathrm{a}$ & 93,12 & $\overline{\mathrm{A} \mathrm{a}}$ \\
\hline Lactofen & 88,85 & $\mathrm{~A}$ a & 64,72 & $\mathrm{~B} \mathrm{~b}$ & 61,33 & $\mathrm{Cb}$ & 71,67 & $\mathrm{~B} \mathrm{~b}$ \\
\hline Chlorimuron-ethyl & 95,10 & $\mathrm{~A}$ a & 67,99 & $\mathrm{~B} \mathrm{~b}$ & 70,37 & $\mathrm{Cb}$ & 87,96 & $\mathrm{~B}$ a \\
\hline Bentazon & 88,40 & A a & 85,97 & $\mathrm{~A}$ a & 80,51 & $\mathrm{~B}$ a & 83,60 & $\mathrm{~B}$ a \\
\hline Bentazon + imazamox & 100,85 & A a & 85,07 & $\mathrm{Ab}$ & 79,40 & $\mathrm{~B} b$ & 82,85 & $\mathrm{~B} \mathrm{~b}$ \\
\hline Imazethapyr & 92,05 & $\mathrm{~A} \mathrm{a}$ & 57,76 & $\mathrm{~B} \mathrm{~b}$ & 45,10 & $\mathrm{D} b$ & 41,97 & $\mathrm{Cb}$ \\
\hline Fomesafen & 87,12 & $\mathrm{~A}$ a & 89,40 & $\mathrm{~A}$ a & 86,92 & $\mathrm{~B}$ a & 87,59 & $\mathrm{~B}$ a \\
\hline Quizalofop-P-ethyl & 86,65 & A a & 90,97 & $\mathrm{~A}$ a & 92,72 & A a & 99,25 & A a \\
\hline Lactofen + quizalofop-P-ethyl & 97,50 & $\mathrm{~A}$ a & 57,65 & $\mathrm{~B} \mathrm{~b}$ & 0,00 & $\mathrm{E} \mathrm{c}$ & 0,00 & $\mathrm{D} \mathrm{c}$ \\
\hline Fomesafen + quizalofop-P-ethyl & 91,53 & $\mathrm{~A}$ a & 88,82 & $\mathrm{~A}$ a & 81,48 & $\mathrm{~B}$ a & 83,52 & $\mathrm{~B}$ a \\
\hline Testemunha & 100,00 & $\mathrm{~A} \mathrm{a}$ & 100,00 & $\mathrm{~A}$ a & 100,00 & A a & 100,00 & $\mathrm{~A} \mathrm{a}$ \\
\hline CV Parcela (\%) & & & & & & & & \\
\hline CV Subparcela (\%) & & & & & & & & \\
\hline
\end{tabular}


Aos 21 DAAH, as plantas tratadas com o imazethapyr apresentaram redução de 58,03\% na altura, enquanto que o chlorimuron-ethyl reduziu em $12,04 \%$. Os herbicidas inibidores da ALS atuam inibindo o crescimento das plantas, e seus efeitos iniciam logo após a aplicação. $\mathrm{O}$ imazethapyr e chlorimuron-ethyl pertençem a grupos químicos diferentes, desta forma a resposta da cultura à ação dessas moléculas ocorre de forma diferente, possivelmente favorecendo que os mecanismos de resistência atuem de forma mais contundente sobre o chlorimuron-ethyl (Vidal e Winkler, 2002).

Agostinetto et al. (2002) avaliaram a resposta da mandioca à aplicação de herbicidas em pós-emergência, e observaram que as plantas tratadas com imazethapyr e chlorimuron-ethyl, apresentaram sintomas de inibição do crescimento, o que ocorreu nas primeiras semanas após as aplicações, sendo que posteriormente houve uma recuperação progressiva.

O número de folhas da mandioca nos tratamentos com haloxyfop, quizalofop-P-ethyl e chlorimuron-ethyl apresentaram resultados semelhantes ao da testemunha durante as avaliações (Tabela 3). Silva et al. (2012a) encontraram resultados similares, em que o haloxyfop e o chlorimuron-ethyl não causaram reduções no número de folhas em relação a testemunha.

Tabela 3. Número de folhas de mandioca, em percentagem relativa à testemunha, aos $0,7,14$ e 21 dias após a aplicação dos tratamentos.

\begin{tabular}{|c|c|c|c|c|c|c|c|c|}
\hline \multirow{3}{*}{ Tratamentos } & \multicolumn{8}{|c|}{ Número de folhas $(\%)$} \\
\hline & \multicolumn{8}{|c|}{ Dias após a aplicação dos herbicidas } \\
\hline & \multicolumn{2}{|l|}{0} & \multicolumn{2}{|c|}{7} & \multicolumn{2}{|c|}{14} & \multicolumn{2}{|c|}{21} \\
\hline Haloxyfop & 97,00 & $\overline{\mathrm{A} a}$ & 94,81 & $\mathrm{~A} \mathrm{a}$ & 90,30 & $\mathrm{~A}$ a & 92,82 & $\mathrm{~A}$ a \\
\hline Lactofen & 92,10 & A a & 29,29 & $\mathrm{Dc}$ & 43,55 & $\mathrm{Db}$ & 46,96 & $\mathrm{Db}$ \\
\hline Chlorimuron-ethyl & 95,00 & A a & 83,90 & $\mathrm{~B}$ a & 85,48 & $\mathrm{~B}$ a & 93,14 & A a \\
\hline Bentazon & 97,20 & A a & 75,86 & $\mathrm{Cb}$ & 79,05 & $\mathrm{~B} \mathrm{~b}$ & 58,69 & $\mathrm{Cc}$ \\
\hline Bentazon + imazamox & 100,00 & A a & 66,67 & $\mathrm{Cc}$ & 63,98 & $\mathrm{Cc}$ & 76,06 & $\mathrm{~B} \mathrm{~b}$ \\
\hline Imazethapyr & 90,80 & A a & 68,97 & $\mathrm{Cb}$ & 59,13 & $\mathrm{Cb}$ & 64,79 & $\mathrm{Cb}$ \\
\hline Fomesafen & 92,04 & A a & 83,32 & $\mathrm{~B}$ a & 83,05 & $\mathrm{~B}$ a & 61,27 & $\mathrm{Cb}$ \\
\hline Quizalofop-P-ethyl & 90,40 & A a & 88,52 & $\mathrm{~B}$ a & 90,60 & A a & 91,42 & A a \\
\hline Lactofen + quizalofop-P-ethyl & 99,10 & A a & 16,65 & $\mathrm{~Eb}$ & 0,00 & $\mathrm{~Eb}$ & 0,00 & E c \\
\hline Fomesafen + quizalofop-P-ethyl & 88,25 & A a & 63,21 & $\mathrm{Cb}$ & 70,97 & $\mathrm{Cb}$ & 64,08 & $\mathrm{Cb}$ \\
\hline Testemunha & 100,00 & A a & 100,00 & $\mathrm{~A} \mathrm{a}$ & 100,00 & A a & 100,00 & A a \\
\hline CV Parcela (\%) & & & & & & & & \\
\hline CV Subparcela (\%) & & & & & & & & \\
\hline
\end{tabular}

$\mathrm{O}$ herbicida bentazon, fomesafen e fomesafen + quizalofop-P-ethyl promoveram necrose nos tecidos foliares, o que provocou a queda e consequente redução do número de folhas, porém não interferiram no surgimento de folhas novas sadias. Efeito similar ocorreu com a aplicação do lactofen e lactofen + quizalofopP-ethyl, entretanto esses tratamentos provocaram danos elevados nas plantas. Silva et al. (2011) constataram que a mistura entre os herbicidas fluazifop-p-butil + fomesafen, promoveu redução na altura da planta e número de folhas, indicando comprometimento do crescimento da mandioca.

A intoxicação das plantas de mandioca foi baixa para os tratamentos haloxyfop e quizalofop-P-ethyl com $5,5 \%$ e $10,0 \%$, aos 3 DAAH e $0,00 \%$ e $5,00 \%$ aos 21 DAAH, respectivamente (Tabela 4). Erasmo et al. (2009) observaram que o haloxyfop foi seletivo a cultura do pinhão manso, não provocando injúrias à cultura. Vários outros autores obtiveram resultados similares, de baixa toxicidade de inibidores da ACCase na cultura 
da mandioca (Abreu, 2010; Silva et al., 2011; Ferreira et al., 2015).

Os valores de intoxicação mais intensos foram observados nos tratamentos lactofen e na mistura lactofen + quizalofop-P-ethyl, desde a primeira avaliação aos 3 DAAH. As folhas rapidamente perderam a turgescência, e se tornaram verdes escuras, ficando posteriormente necróticas. O lactofen aplicado isolado não interrompeu a emissão de folhas novas, mas retardou o desenvolvimento e aos 21 DAAH as plantas estavam debilitadas e apresentaram sintomas de intoxicação de $38,50 \%$ (Tabela 4). Tais resultados são similares aos relatados por Silva et al. (2012a), em que o lactofen foi um dos tratamentos que promoveu maiores níveis de intoxicação a cultura da mandioca.

Tabela 4. Intoxicação de plantas de mandioca aos 3, 6, 9, 12, 15, 18, 21 dias após a aplicação (DAAH) de herbicidas.

\begin{tabular}{|c|c|c|c|c|c|c|c|c|c|c|}
\hline \multirow{3}{*}{ Tratamentos } & \multicolumn{10}{|c|}{ Nível de intoxicação de plantas (\%) } \\
\hline & \multicolumn{10}{|c|}{ Dias após a aplicação dos herbicidas } \\
\hline & 3 & 6 & & 9 & & 12 & & 15 & 18 & 21 \\
\hline Haloxyfop & $5,50 \mathrm{Aa}$ & 4,00 & $\mathrm{Aa}$ & 2,75 & $\mathrm{Aa}$ & 2,00 & $\mathrm{Aa}$ & $2,50 \mathrm{Aa}$ & $1,75 \mathrm{Aa}$ & $0,00 \mathrm{Aa}$ \\
\hline Lactofen & $75,50 \mathrm{Gd}$ & 83,75 & $\mathrm{Ge}$ & 66,25 & $\mathrm{Fc}$ & 62,75 & Ec & $50,25 \mathrm{Db}$ & $45,75 \mathrm{Db}$ & $38,50 \mathrm{Da}$ \\
\hline Chlorimuron-ethyl & $12,00 \mathrm{Ba}$ & 23,50 & $\mathrm{Cb}$ & 20,75 & $\mathrm{Cb}$ & 16,75 & $\mathrm{Ba}$ & $13,75 \mathrm{Bb}$ & $13,00 \mathrm{Ba}$ & $10,00 \mathrm{Ba}$ \\
\hline Bentazon & $47,50 \mathrm{Ed}$ & 36,75 & Dc & 35,75 & Dc & 27,00 & $\mathrm{Cb}$ & $17,25 \mathrm{Ba}$ & $15,50 \mathrm{Ca}$ & $14,50 \mathrm{Ba}$ \\
\hline Bentazon + imazamox & $41,25 \mathrm{Da}$ & 44,75 & $\mathrm{Ea}$ & 50,00 & $\mathrm{~Eb}$ & 54,50 & $\mathrm{Db}$ & $60,25 \mathrm{Ed}$ & $63,75 \mathrm{Ec}$ & $67,50 \mathrm{Ec}$ \\
\hline Imaz & $18,25 \mathrm{Ca}$ & 20,00 & $\mathrm{Ca}$ & 24,50 & $\mathrm{Cb}$ & 28,50 & $\mathrm{Cb}$ & $35,25 \mathrm{Cc}$ & $39,75 \mathrm{Cc}$ & $40,50 \mathrm{Dd}$ \\
\hline Fome & $40,00 \mathrm{Da}$ & 44,50 & $\mathrm{Ea}$ & 49,50 & $\mathrm{~Eb}$ & 57,00 & Dc & $52,00 \mathrm{Db}$ & $45,75 \mathrm{Db}$ & $30,25 \mathrm{Ca}$ \\
\hline Quizalofop-P-€ & $8,00 \mathrm{Bb}$ & 10,00 & $\mathrm{Bb}$ & 10,00 & $\mathrm{Bb}$ & 8,50 & $\mathrm{Bb}$ & $8,00 \mathrm{Bb}$ & $5,25 \mathrm{Ab}$ & $5,00 \mathrm{Aa}$ \\
\hline Lactofen + quizalofop-P-c & $86,75 \mathrm{Ha}$ & 95,50 & $\mathrm{Hb}$ & 98,50 & $\mathrm{~Gb}$ & 100,00 & $\mathrm{Fb}$ & $100,00 \mathrm{Fa}$ & $100,00 \mathrm{Fa}$ & $100,00 \mathrm{Fa}$ \\
\hline Fomesafen + quizalofop-I & $67,75 \mathrm{Fc}$ & 67,00 & $\mathrm{Fc}$ & 65,25 & $\mathrm{Fc}$ & 63,75 & Ec & $48,25 \mathrm{Db}$ & $42,50 \mathrm{Cb}$ & $27,50 \mathrm{Ca}$ \\
\hline CV Parcela $(\%)$ & & & & & & $\overline{12,91}$ & & & & \\
\hline CV Subparcela (\%) & & & & & & 8,16 & & & & \\
\hline
\end{tabular}

A mistura lactofen + quizalofop-P-ethyl ocasionou a morte das plantas antes do término das avaliações, demonstrando a incompatibilidade da mistura entre herbicidas com as moléculas quizalofop-P-ethyl e lactofen, para a cultura da mandioca.

Os tratamentos fomesafen e a mistura fomesafen + quizalofop-P-ethyl, promoveram danos elevados inicialmente com sintomas de clorose e necrose das áreas tratadas (folhas e haste) e perda de turgescência foliar, similar ao efeito observado com o lactofen, entretanto, as plantas apresentaram rápida recuperação.

Silveira et al. (2012), ao avaliarem as características fotossintéticas de cultivares de mandioca tratadas a mistura comercial fluazifop-p-butyl + fomesafen, relataram que ocorreu aumento no consumo de $\mathrm{CO}_{2}$, indicando uma aceleração do metabolismo após a aplicação do formulado. Silva et al. (2011) descreveram que após a aplicação de fluazifopp-butyl + fomesafen, as plantas apresentaram intensa desfolha mas com recuperação após o surgimento de novas folhas sem sintomas de intoxicação.

$\mathrm{O}$ uso do herbicida formulado bentazon + imazamox apresentou intoxicação inicial de $41,25 \%$, com sintomas de branqueamento das folhas (Tabela 4). Os sintomas foram progressivos e se intensificaram até aos 21 DAAH com intoxicação de 67,50\%. Observouse que as novas folhas apresentavam má formação, lóbulos deformados e amarelados. Inoue et al. (2014) relataram que o uso do bentazon + imazamox, em plantas de pinhão manso, apresentou elevados índices de intoxicação durante as avaliações, assim como redução na altura e diâmetro do caule na última avaliação aos 35 DAAH. 
O chlorimuron-ethyl apresentou pouca intoxicação sobre as plantas de mandioca, durante as avaliações, o que reforça o fato de que as plantas da cv. Aciolina podem apresentar tolerância à ação desses herbicidas, ocorrendo um decréscimo nos níveis de intoxicação da cultura dos 6 DAAH $(23,50 \%)$ aos 21 DAAH $(10,0 \%)$. Os sintomas verificados foram de leve clorose nas folhas mais velhas. A baixa toxicidade do chlorimuron-ethyl em plantas de mandioca foi relatada por Silva et al. (2012a) e Moreira (2014), em que foi observado leve intoxicação aos 7 DAAH, havendo tendência de redução dos sintomas, ao longo das avaliações.

A intoxicação causada pelo imazethapyr foi de 18,25\% aos 3 DAAH, com o decorrer do ciclo as folhas mais jovens apresentavam-se com clorose, aos 21 DAAH, com as plantas pouco desenvolvidas e intoxicação de 40,50\%. Agostinetto et al. (2002) observaram valores elevados de toxicidade em plantas de mandioca aos 8 e 14 DAAH com imazethapyr e chlorimuron-ethyl, promovendo média superior a 50\% de injúria sobre as plantas de mandioca, entretanto ambos apresentaram recuperação ao final das avaliações. Os sintomas relatados por esses autores são similares aos observados com o uso do imazethapyr, com inibição do crescimento e clorose foliar na região meristemática.

O acúmulo de massa seca das folhas (MSF) e massa seca da haste (MSH) e massa seca das raízes (MSR), massa seca total (MST) e área foliar das plantas tratadas com haloxyfop, quizalofop-P-ethyl e chlorimuron-ethyl não diferiram da testemunha (Tabela 5). O chlorimuron-ethyl não promoveu danos significativos sobre as variáveis avaliadas, os resultados são promissores tendo em vista de se tratar de um herbicida indicado para o controle de dicotiledôneas, aplicado em pós-emergência.

Tabela 5. Massa seca foliar (MSF), da haste (MSH), de raízes (MSR), massa seca total (MST) e área foliar $(\mathrm{AF})$ da mandioca, em porcentagem relativa à testemunha, aos 21 dias após a aplicação dos herbicidas.

\begin{tabular}{|c|c|c|c|c|c|c|c|c|c|c|}
\hline Tratamentos & MSF & & MSH & & MSR & & MST & & $\mathrm{AF}$ & \\
\hline Haloxyfop & 96,42 & $\mathrm{a}$ & 103,51 & $\mathrm{a}$ & 118,44 & $\mathrm{a}$ & 101,82 & $\mathrm{a}$ & 99,33 & $\mathrm{a}$ \\
\hline Lactofen & 42,69 & $\mathrm{~d}$ & 45,91 & c & 37,98 & $\mathrm{~b}$ & 43,58 & $\mathrm{~d}$ & 59,97 & $\mathrm{c}$ \\
\hline Chlorimuron-ethyl & 92,34 & $\mathrm{a}$ & 97,34 & $\mathrm{a}$ & 118,19 & $\mathrm{a}$ & 97,36 & $\mathrm{a}$ & 96,76 & $\mathrm{a}$ \\
\hline Bentazon & 78,45 & $\mathrm{~b}$ & 88,73 & $\mathrm{a}$ & 80,71 & $\mathrm{a}$ & 82,96 & $\mathrm{~b}$ & 91,29 & $\mathrm{a}$ \\
\hline Bentazon + imazamox & 56,67 & $\mathrm{c}$ & 72,06 & $\mathrm{~b}$ & 59,64 & $\mathrm{~b}$ & 63,39 & c & 48,48 & $\mathrm{c}$ \\
\hline Imazethapyr & 62,92 & $\mathrm{c}$ & 66,42 & $\mathrm{~b}$ & 60,83 & $\mathrm{~b}$ & 64,13 & c & 54,11 & c \\
\hline Fomesafen & 73,15 & $\mathrm{~b}$ & 84,07 & $\mathrm{~b}$ & 61,42 & $\mathrm{~b}$ & 76,34 & b & 84,88 & $\mathrm{~b}$ \\
\hline Quizalofop-P-ethyl & 95,85 & $\mathrm{a}$ & 98,53 & $\mathrm{a}$ & 96,44 & $\mathrm{a}$ & 97,03 & $\mathrm{a}$ & 99,19 & $\mathrm{a}$ \\
\hline Lactofen + quizalofop-P-ethyl & 0,00 & $\mathrm{e}$ & 0,00 & $\mathrm{~d}$ & 0,00 & $\mathrm{c}$ & 0,00 & $\mathrm{e}$ & 0,00 & $\mathrm{~d}$ \\
\hline Fomesafen + quizalofop-P-ethyl & 73,94 & $\mathrm{~b}$ & 80,39 & $\mathrm{~b}$ & 66,77 & $\mathrm{~b}$ & 75,79 & $\mathrm{~b}$ & 71,24 & $\mathrm{~b}$ \\
\hline Testemunha & 100,00 & $\mathrm{a}$ & 100,00 & a & 100,00 & $\mathrm{a}$ & 100,00 & $\mathrm{a}$ & 100,00 & $\mathrm{a}$ \\
\hline $\mathrm{CV}(\%)$ & 10,67 & & 15,64 & & 35,14 & & 9,56 & & 13,66 & \\
\hline
\end{tabular}

Os resultados apontam que os inibidores da ACCase não causaram danos a cultura. Tais herbicidas são indicados para o controle de monocotiledôneas, o que justifica a tolerância da cultura. Abreu et al. (2010) descreveram que o uso do herbicida haloxyfop reduziu significativamente a porcentagem de raízes comerciais de mandioca da variedade IAC14, enquanto os tratamentos fluazifop-p-butil e quizalofop-p-ethyl promoveram valores similares ao da testemunha. Entretanto, o mesmo haloxyfop apresentou valores de porcentagem de raízes comerciais elevados, superiores aos demais tratamentos quando aplicado na variedade IAC 576-70.

Moreira (2014) observou que o chlorimuron-ethyl e fluazifop-p-butil, apresentaram valores similares na massa seca 
total para a cultura da mandioca. Entretanto, há relatos na literatura da sensibilidade da mandioca ao chlorimuron-ethyl. Agostinetto et al. (2002) e Silva et al. (2012a) concluíram que esse herbicida é pouco seletivo para a cultura. $\mathrm{O}$ primeiro autor observou que o chlorimuronethyl reduziu a massa seca da parte aérea em $38,9 \%$ aos 28 DAAH e o segundo observou redução de $34,75 \%$ para a massa seca total aos 35 DAAH.

Os tratamentos bentazon, fomesafen e fomesafen + quizalofop-P-ethyl, apresentaram valores intermediários para o acúmulo de massa seca e área foliar. Embora os resultados tenham diferido da testemunha, são resultados promissores, tendo em vista o ciclo da cultura e a tendência à recuperação. Esses tratamentos promoveram a queda das folhas, entretanto a cultura apresentou boa recuperação, com a emissão de novas folhas.

Valores elevados de massa seca da parte aérea e massa seca total foram relatados com o uso do bentazon aplicado na mandioca por Agostineto et al. (2002) e Silva et al. (2012a). Assim como valores intermediários de massa seca em relação a testemunha foram observados pelos mesmos autores com o uso do fomesafen. Erasmo et al. (2009) relatam que o fomesafen, reduziu significativamente o acúmulo de massa seca das raízes da cultura do pinhão manso em condição de campo.

Abreu (2010) relata que os herbicidas fomesafen e bentazon reduziram significativamente a produtividade de raiz e massa seca da raiz no final do ciclo da cultura para as cultivares IAC14 e IAC576-70. Agostinetto et al. (2002) concluíram que os herbicidas fomesafen e bentazon, embora tenham causado elevado grau de injúria nas duas primeiras semanas após aplicação, não provocam danos permanentes possibilitando a recuperação das plantas, considerando assim tais herbicidas como potenciais para utilização na cultura da mandioca.

O imazethapyr e a mistura bentazon + imazamox reduziram significativamente as variáveis de massa seca e área foliar da mandioca. O lactofen assim como a mistura lactofen + quizalofop-P-ethyl promoveram danos irreversíveis a cultura da mandioca. Demonstrando a impossibilidade de uso em pósemergência destes herbicidas em plantas de mandioca cv. Aciolina.

Portanto, o presente trabalho mostra que a cv. Aciolina apresentou resultados promissores quanto à tolerância aos herbicidas haloxyfop e quizalofop-P-ethyl, o que possibilita a inclusão das moléculas para o controle de plantas daninhas de folha estreita. Os herbicidas chlorimuron-ethyl, bentazon, fomesafen e a mistura fomesafen + quizalofopP-ethyl, apresentaram bons resultados para a utilização no controle de plantas daninhas aumentando a possibilidade de controle de espécies mono e dicotiledôneas em pósemergência.

\section{Conclusões}

As plantas de mandioca, cultivar Aciolina, apresentam elevada tolerância aos herbicidas haloxyfop e quizalofop-P-ethyl e chlorimuron-ethyl em aplicações pósemergentes.

Os herbicidas imazethapyr, lactofen e o formulado bentazon + imazamox provocam danos severos ao desenvolvimento da cultura da mandioca.

A mistura lactofen + quizalofop-P-ethyl potencializou o efeito dos herbicidas sobre a cultura da mandioca, ocasionando a morte das plantas.

\section{Referências}

Abreu, M.L. Seletividade de herbicidas à cultura da mandioca. 2010. 65 f. Dissertação (Mestrado em Agronomia) - Faculdade de Ciências Agronômicas, Universidade Estadual Paulista, Botucatu, 2010.

Abreu, M.L; Bicudo, S.J; Curcelli, F; Aguiar, F.B; Brachtvogel, E.L. Efeito de diferentes herbicidas aplicados na cultura da mandioca na quantidade qualidade de raízes comerciais. 
Revista Raízes e Amidos Tropicais, v.6, n.1, p.66-76, 2010.

Agostinetto, D; Fleck, N.G; Rizzardi, M.A; Thomas, A.L. Seletividade de herbicidas latifolicidas aplicados à mandioca em pósemergência. Revista Brasileira de Herbicidas, v.3, n.l, p.39-43, 2002.

Albuquerque, J.A.A.; Sediyama, T; Silva, A.A; Carneiro, J.E.S.; Cecon, P.R.; Alves, J.M.A. Interferência de plantas daninhas sobre a produtividade da mandioca (Manihot esculenta). Planta Daninha, v.26, n.2, p.279289, 2008.

Albuquerque, J.A.A; Evangelista, M.O; Mates, A.P.K; Alves, J.M.A; Oliveira, N.T; Sediyama, T; Silva, A.A. Occurrence of weeds in Cassava savanna plantations in Roraima. Planta Daninha, v.32, n.1, p.91-98, 2014.

Biffe, D.F; Constantin, J; Oliveira Jr., R.S; Rios, F.A; Franchini, L.H.M.; Gemelli, A. et al. Avaliação de herbicidas para dois cultivares de mandioca. Planta Daninha, v.28, n.4, p.807816, 2010.

Brasil. Ministério da Agricultura, Pecuária e Abastecimento. Coordenação Geral de Agrotóxicos e Afins. Disponível em $<$ http://agrofit.agricultura.gov.br/agrofit_cons/ principal_agrofit_cons $>$. Acesso em: $10 \mathrm{fev}$. 2016

Cardoso, A.D.; Viana, A.E.S.; Barbosa, R.P.; Teixeira, P.R.G.; Cardoso Júnior, N.S.; Fogaça, J.J.N.L. Levantamento fitossociológico de plantas daninhas na cultura da mandioca em vitória da conquista, Bahia. Bioscience Journal, v.29, n.5, p.1130-1140, 2013.

Erasmo, E.A.L; Costa, N.V; Terra, M.A; Fidelis, R.R. Tolerância inicial de plantas de pinhão-manso a herbicidas aplicados em pré e pós-emergência. Planta Daninha, v.27, n.3, p.571-580, 2009.

Ferreira, E.A; Matos, C.C; Barbosa, E.A; Silva, D.V; Santos, J.B; Pereira, G.A.M. et al. Respostas fisiológicas da mandioca à aplicação de herbicidas. Semina: Ciências Agrárias, v.36, n.2, p.645-656, 2015.

Inoue, M.H; Pereira, K.M; Mendes, K.F; Santos, S.G; Dallacort, R; Possamai, A.C.S. Seletividade de herbicidas para pinhão manso em condições de casa de vegetação e campo. Bioscience Journal, v.30, n.2, p.791-801, 2014.

Johanns, O; Contiero, R.L. Efeitos de diferentes períodos de controle e convivência de plantas daninhas com a cultura da mandioca. Revista Ciência Agronômica, v.37, n.3, p.326-331, 2006.

Machado, A.F.L.; Camargo, A.P.M.; Ferreira, L.R.; Sediyama, T.; Ferreira, F.A.; Viana, R.G. Misturas de herbicidas no manejo de plantas daninhas na cultura do feijão. Planta Daninha, v.24, n.1, p.107-114, 2006.

Moreira, E.S. Seletividade e épocas de aplicação de herbicidas em Mandioca. 2014. 62 f. Dissertação (mestrado em agronomia) Universidade Estadual do Sudoeste da Bahia, Vitória da Conquista, 2014.

Moura, G.M. Interferência de plantas daninhas na cultura da mandioca (Manihot esculenta Crantz) no Estado do Acre. Planta Daninha, v.18, n.3, p.451-456, 2000.

Nunes, L.B.; Santos, W.J.; Cruz, R.S. Rendimento de extração e caracterização química e funcional de féculas de mandioca da região do semi-árido baiano. Alimento e Nutrição, v.20, n.1, p.129-134, 2009.

Schons, A.; Streck, N.A.; Storck, L.; Buriol, G.A.; Zanon, A.J.; Pinheiro, D.G.; Kraulich, B. Arranjos de plantas de mandioca e milho em cultivo solteiro e consorciado: crescimento, desenvolvimento e produtividade. Bragantia, v.68, n.1, p.155-167, 2009.

Silva, D.V; Santos, J.B; Silveira, H.M; Carvalho, F.P; Castro Neto, M.D; Ferreira, E.A. et al. Tolerância de cultivares de mandioca aos herbicidas fomesafen e fluazifop-pbutil. Revista Brasileira de Herbicidas, v.10, n.3, p.219-231, 2011. 
Silva, D.V; Santos, J.B.; Carvalho, F.P.; Ferreira, E. A.; França, A. C.; Fernandes, J.S.C. et al. Seletividade de herbicidas pós-emergentes na cultura da Mandioca. Planta Daninha, v.30, n.4, p.835-841, 2012a.

Silva, D.V.; Santos, J.B.; Ferreira, E.A; Silva, A.A.; França, A.C.; Sediyama, T. Manejo de plantas daninhas na cultura da mandioca. Planta Daninha, v.30, n.4, p.901-910, 2012 b.

Silva, F.M.L.; Abreu, M.L.; Brachtvogel, E.L.; Curcelli, F.; Gimenes, M.J.; Lara, A.C.C. Moléculas de herbicidas seletivos à cultura da mandioca. Revista Trópica - Ciências Agrárias e Biológicas, v.3, n.2, p.61-72, 2009.

Silveira, H.M; Silva, D.V; Carvalho, F.P; Castro Neto, M.D; Silva, A.A; Sediyama, T. Características fotossintéticas de cultivares de mandioca tratadas com fluazifop-p-butyl e fomesafen. Revista Agro@mbiente On-line, v.6, n.3, p.222-227, 2012.

Sociedade Brasileira Da Ciência Das Plantas Daninhas (SBCPD). Procedimentos para instalação, avaliação e análise de experimentos com herbicidas. Londrina: SBCPD, 1995.

Vidal, R.A.; Winkler, L.M. Resistência de plantas daninhas: seleção ou indução à mutação pelos herbicidas inibidores de acetolactato sintase (ALS). Pesticidas: Revista de Ecotoxicologia e Meio Ambiente, v.12, n.1, p.31-42, 2002. 